\title{
Optimisation robuste multi-niveaux et multi-objectif de structures mécaniques complexes
}

\author{
Samir Ghanmi ${ }^{1, a}$, Mohamed Guedri ${ }^{1}$, Mohamed Lamjed Bouazizi ${ }^{1}$ \\ ET Noureddine Bouhaddi ${ }^{2}$ \\ 1 Institut Préparatoire aux Études d'Ingénieur de Nabeul (IPEIN), Campus universitaire M'rezka 8000, Tunisie \\ 2 Laboratoire de Mécanique Appliquée R. Chaléat, UMR 6604 CNRS, 24 chemin de l'Épitaphe, 25000 Besançon, \\ France
}

Reçu le 29 juin 2009, accepté le 23 juin 2010

\begin{abstract}
Résumé - Cet article présente une nouvelle approche d'optimisation multi-objectifs robuste, et multiniveaux, pour la conception des structures mécaniques complexes. Cette optimisation est à deux niveaux : système et élément. Le problème multi-objectifs robuste, au niveau système, à résoudre est à quatre fonctions coût : d'une part la minimisation de la masse globale et le déplacement en un point fixé de la structure mécanique étudiée, d'autre part la maximisation des robustesses respectivement de la masse et du déplacement alors que le problème multi-objectifs robuste au niveau élément est à deux fonctions coût : la minimisation de la masse de l'élément et la maximisation de sa robustesse. Contrairement aux formulations existantes, cette nouvelle approche tient compte des incertitudes sur les paramètres de conception tant au niveau système qu'au niveau élément. De plus, elle répond au partage des tâches pratiqué dans l'industrie.
\end{abstract}

Mots clés : Optimisation multi-objectif / optimisation multi-niveaux / incertitudes / conception robuste / algorithme génétique

\begin{abstract}
Robust multi-level and multi-objective optimization of complex mechanical structures. This paper presents a new approach of robust multi-objective optimization and multilevel for design of complex mechanical structures. This optimization is on two levels: system and elements. The robust multi-objective problem on system-level to be solved is with four cost functions: on the one hand the minimization of the global mass and displacement in a fixed point of mechanical structure to be studied, and on the other hand the maximization of the robustness respectively of the mass and displacement whereas the robust multi-objective problem on the element-level is with two cost functions: minimization of the element mass and maximization of its robustness. Contrary to the existing formulations, this new approach takes into account uncertainties on the design parameters on the system-level and on the element-level. Moreover, it answers the task sharing commonly used in industry field.
\end{abstract}

Key words: Multi-objective optimization / multi-level optimization / uncertainties / robust design / genetic algorithm

\section{Introduction}

La conception des structures complexes (de grande taille) telles que les structures aéronautiques, aérospatiales, etc. ne peut être accomplie sans application des méthodes d'optimisation. La taille du problème d'optimisation dépend à la fois du nombre de variables et des contraintes de conception exigées, en particulier pour la conception d'une structure avec des interactions fortes entre ses divers composants structuraux.

\footnotetext{
a Auteur pour correspondance :

samir.ghanmi@ipein.rnu.tn
}

Le traitement des problèmes d'optimisation par les procédures conventionnelles, où tous les composants structuraux sont considérés simultanément (système global), mène à des temps de calcul prohibitifs qui dépassent parfois les délais fixés par le cahier des charges. Pour pallier ce problème, plusieurs schémas d'optimisation multi-niveaux ont été proposés au cours de ces dernières années [1-3]. Ces techniques proposent alors de découper le problème original en un problème d'optimisation au niveau «système », travaillant sur des variables de niveau global, couplé à plusieurs problèmes d'optimisations travaillant au niveau « élément » sur quelques variables 
détaillées à la fois. Chaque problème peut être optimisé indépendamment.

Pour les applications structurales, la décomposition d'un problème de conception peut être basée sur la décomposition d'une structure globale en sous-structures.

Les variables de système (structure globale) sont des quantités globales qui affectent le système et les sous-systèmes, et les contraintes du système sont des contraintes globales, comme le déplacement structural, etc. Les variables de sous-systèmes sont, par exemple, des dimensions locales. L'optimisation multi-niveaux procède en réitérant dans les deux sens entre la solution du système et les sous-systèmes, alors que les contraintes locales peuvent être, par exemple, des niveaux de contrainte ou une résistance au flambement.

Dans la littérature, l'utilisation de l'optimisation multi-objectifs et multi-niveaux [1-3] est généralement confrontée aux difficultés suivantes :

- la transformation du problème multi-objectif au niveau système en un autre mono-objectif : cette transformation exige la connaissance a priori du poids de chaque fonction coût par rapport à l'autre. En pratique, cette tâche n'est pas toujours facile et disponible, soit par manque d'expériences ou d'informations, soit parce que les fonctions coût sont non commensurables. De plus, lorsque l'espace de conception n'est pas convexe, la méthode de pondération ignore la partie non convexe de l'ensemble Pareto optimal; or cette partie des solutions représente un bon compromis ;

- la présence des incertitudes sur les paramètres de conception : en effet, il est bien connu qu'une solution théoriquement excellente peut s'avérer catastrophique en pratique si les erreurs commises, lors de la fabrication par exemple, ne permettent pas d'obtenir les valeurs des variables de conception avec une précision suffisante : même un faible écart par rapport à leur valeur optimale théorique pourra se traduire par un comportement très éloigné de celui prévu par l'optimisation théorique. Une solution sous-optimale mais stable vis-à-vis des incertitudes sera alors beaucoup plus intéressante pour le concepteur.

Afin de résoudre le problème multi-objectif et multiniveaux, plusieurs algorithmes ont été utilisés et en particulier les algorithmes évolutionnaires de type algorithme génétique au niveau système ou bien au niveau élément [1], ou de type gradient [2], ou encore des codes de calcul spécifiques à un domaine particulier comme les codes « AISC-LRFD, AISC et GBJ17-88 », utilisés par [3] pour optimiser le comportement d'une structure mécanique en acier.

Ce papier présente une contribution portant sur l'optimisation multi-objectif et multi-niveaux. Le problème d'optimisation initial est décomposé en un problème d'optimisation au niveau système, travaillant sur des variables globales qui permettent de modéliser le comportement global du système, et plusieurs problèmes d'optimisation locaux travaillant sur des variables de conception locales, représentant le comportement local de chaque élément. La méthode proposée tient compte de la présence des incertitudes sur les paramètres de conception aussi bien au niveau système qu'au niveau des éléments. La prise en compte de ces incertitudes, dans les procédures d'optimisation multi-objectifs, a nécessité l'introduction d'indicateurs de robustesse des solutions vis-à-vis de ces incertitudes afin de trouver les solutions les plus stables.

La prise en compte des incertitudes, lors de la conception, a fait l'objet de plusieurs études ces dernières années dont on cite les travaux $[4,5]$. Ces derniers ont proposé d'intégrer un critère de robustesse au cours de la procédure d'optimisation. Ce critère est formulé par l'emploi d'une pondération de la moyenne et l'écart type de la fonction objectif. Ainsi, on est ramené à un problème d'optimisation mono-objectif robuste à minimiser. Le principal inconvénient de cette approche réside dans l'emploi de la méthode de Monte Carlo. En effet, le coût de calcul nécessaire pour l'évaluation de la moyenne et l'écart type de la fonction objectif à son optimum peut être prohibitif. Pour y remédier, Ghanmi et al. [6] ont proposé d'intégrer le critère de robustesse comme une fonction coût supplémentaire et le problème monoobjectif s'est transformé en un autre problème multiobjectifs. Ils ont proposé l'emploi de la méthode des surfaces de réponse stochastiques SRSM [7] qui permet de remplacer le modèle éléments finis (MEF) complexe par un polynôme plus simple à gérer. Cette méthode a permis de réduire le nombre de simulations exigé pour l'évaluation adéquate des incertitudes, d'analyser les incertitudes systématiques et de fournir les niveaux de confiances estimés.

La résolution du problème multi-objectifs et multiniveaux est basée sur l'utilisation d'un algorithme génétique $(\mathrm{AG})$ multi-niveaux. Le nombre d'AGs utile dépend du nombre de familles d'éléments dans chaque niveau d'optimisation. Après optimisation, les solutions obtenues sont optimales et robustes vis-à-vis des incertitudes sur les paramètres de conception.

Le papier est organisé en deux parties. Dans la première partie nous avons présenté la méthodologie proposée. Celle-ci contient le critère de robustesse utilisé lors de l'optimisation et la manière avec laquelle on transforme un problème multi-objectifs en un autre problème multiobjectifs robuste et multi-niveaux. Dans la seconde partie, nous avons appliqué cette méthodologie sur un exemple simple afin de mieux comprendre son application et voir son intérêt pour le concepteur dans une phase d'avantprojet ou de projet.

\section{Optimisation multi-objectifs robuste et multi-niveaux}

D’une façon générale, un problème d'optimisation multi-objectifs est exprimé par l'équation suivante :

$$
\left\{\begin{array}{c}
\min F(x)=\left(f_{1}(x), f_{2}(x), \ldots, f_{k}(x)\right)^{T} \\
x \in S
\end{array}\right.
$$


où : $f_{1}(x), f_{2}(x), \ldots, f_{k}(x)$ sont des fonctions coût, $\left(x_{1}, x_{2}, \ldots, x_{n}\right)^{T}$ est le vecteur des $n$ paramètres d'optimisation, $S \in R^{n}$ représente l'ensemble des solutions réalisables et $F(x)$ est le vecteur des fonctions à optimiser. L'ensemble des solutions Pareto optimales sont celles qui ne sont pas dominées par d'autres solutions.

Si on considère un problème de minimisation où $y, z \in$ $S$ sont deux vecteurs de $n$ paramètres d'optimisation, $y$ domine $z$ noté $y>z$ si et seulement si $\forall i \in[1, \ldots, n]$ : $f_{i}(y) \leq f_{i}(z)$ et $\exists j \in[1, \ldots, n]: f_{j}(y)<f_{j}(z)$. Pour la recherche des solutions optimales, nous exploitons les algorithmes génétiques (NSGA : Non-dominated Sorting Genetic Algorithm) [7] qui permettent de mieux explorer l'espace de conception et de couvrir l'ensemble du front de Pareto optimal.

\subsection{Optimisation multi-objectifs robuste}

Pour étudier le comportement des systèmes mécaniques présentant des incertitudes sur leurs paramètres de conception, le concepteur doit tenir compte de la robustesse vis-à-vis de ces incertitudes, afin de garantir une conception robuste et performante, car il est bien connu qu'une solution, théoriquement excellente, peut s'avérer catastrophique en pratique si les erreurs commises lors de la fabrication, par exemple, ne permettent pas d'obtenir les valeurs des variables de conception avec une précision suffisante : même un faible écart par rapport à leur valeur optimale théorique pourra se traduire par un comportement très éloigné de celui prévu par le concepteur. Une solution sous-optimale mais stable vis-àvis des incertitudes sera alors beaucoup plus intéressante pour le concepteur. Lors de l'optimisation, la prise en compte des incertitudes sur les paramètres de conception nécessite l'introduction d'indicateurs de robustesse des solutions optimales vis-à-vis de ces incertitudes. Le problème d'optimisation robuste est construit en optimisant, simultanément, les fonctions coût initiales et leurs robustesses : si le problème d'optimisation multi-objectif initial est défini par l'équation (1), alors, le problème d'optimisation multi-objectif robuste est posé de la manière suivante [8] :

$$
\left\{\begin{array}{l}
\min F(x)=\left(f_{1}(x), f_{1}^{v}(x), \ldots, f_{m}(x), f_{m}^{v}(x)\right)^{T} \\
x \in S
\end{array}\right.
$$

où :

$$
f_{i}^{v}(x)=\left(\sigma_{f_{i}} / \mu_{f_{i}}\right)=\frac{1}{f_{i}^{r}(x)}
$$

et $f_{i}^{v}(x)$ et $f_{i}^{r}(x)$ sont respectivement les fonctions vulnérabilité et robustesse de la même fonction coût $f_{i}(x)$, données par l'équation (3). Le rapport $\sigma_{f_{i}} / \mu_{f_{i}}$ désigne la dispersion. $\mu_{f_{i}}$ et $\sigma_{f_{i}}$ sont respectivement la moyenne et l'écart type calculés sur l'ensemble des échantillons $\left(f_{i}\right)_{1<i<N}(x)$ de $f(x)$. « $N$ » représente le nombre de simulations par la méthode d'Hyper Cube Latin [9].

\subsection{Optimisation multi-niveaux}

Le problème d'optimisation multi-objectifs robuste et multi-niveaux est posé comme suit : on considère une structure mécanique complexe composée de l'assemblage de « $\mathrm{K} »$ éléments (sous-structures). La géométrie détaillée de l'élément $i »$ est définie par le vecteur des variables locales associées $X=X_{i / i=1 . . K}=\left(x_{i, j}\right)_{j=1, \ldots, n l_{i}}$ et les variables globales associées sont notées $Y=$ $Y_{i / i=1 . . K}=\left(y_{i, j}\right)_{j=1, \ldots, n g_{i}}$. Il existe de plus une fonction $f: \Re^{n l_{i}} \rightarrow \Re^{n g_{i}}$ telle que $: Y_{i}=f\left(X_{i}\right)$.

Pour l'optimisation au niveau système, le problème multi-objectif robuste est défini par l'équation :

$$
\left\{\begin{array}{c}
\min F(Y)=\left(f_{1}(Y), f_{1}^{v}(Y), ., f_{m}(Y), f_{m}^{v}(Y)\right)^{T} \\
\text { sous contraintes } G_{j}(Y) \leq 0 ; j=1, \ldots, n_{s} \\
Y_{\min } \leq Y \leq Y_{\operatorname{Max}}
\end{array}\right.
$$

Pour l'optimisation au niveau élément (pour le $i$ ème élément), le problème multi-objectif robuste est défini par l'équation suivante :

$$
\left\{\begin{array}{c}
\min F_{i}(X)=\left(f_{i_{1}}(X), f_{i_{1}}^{v}(X), ., f_{i_{m}}(X), f_{i_{m}}^{v}(X)\right)^{T} \\
\text { sous contraintes (s.c) } G_{i}\left(Y^{*}, X\right) \leq 0 \\
G_{j}(X) \leq 0 ; j=1, \ldots, n_{i} \\
X_{\min } \leq X \leq X_{\mathrm{Max}}
\end{array}\right.
$$

où $F_{i}(X)$ est le vecteur des fonctions coût et leurs vulnérabilités de l'élément $« i^{*}, G_{j}(X)$ est la contrainte et $n_{i}$ est le nombre des contraintes; $G_{i}\left(Y^{*}, X\right)$ est la contrainte relative additionnelle d'égalité qui relie des variables de conception des niveaux et simplifie l'accouplement entre eux.

L'exposant $(*)$ dénote les variables dont la valeur est fixée au niveau considéré, égale à la valeur sortie des optimisations de l'autre niveau.

L'application de la méthodologie proposée nécessite :

- au niveau local, des paramètres de conception considérés comme locaux, principalement de type géométrique et qui sont propres à l'élément ou sousstructure;

- au niveau global, des paramètres de conception, considérés comme globaux, généralement les propriétés des matériaux de la structure, comme : module d'élasticité longitudinal $(E)$, masse volumique $(\rho)$, etc.;

- pour la connexion entre les sous-structures, sont exigées dès le départ la (les) condition (s) de connexion AGs comme contrainte dans les problèmes d'optimisation locaux.

Pour résoudre le problème multi-niveaux, un algorithme génétique multi-niveaux est proposé (Fig. 1). Le nombre d'AGs utile dépend du nombre de familles d'éléments dans chaque niveau d'optimisation. 


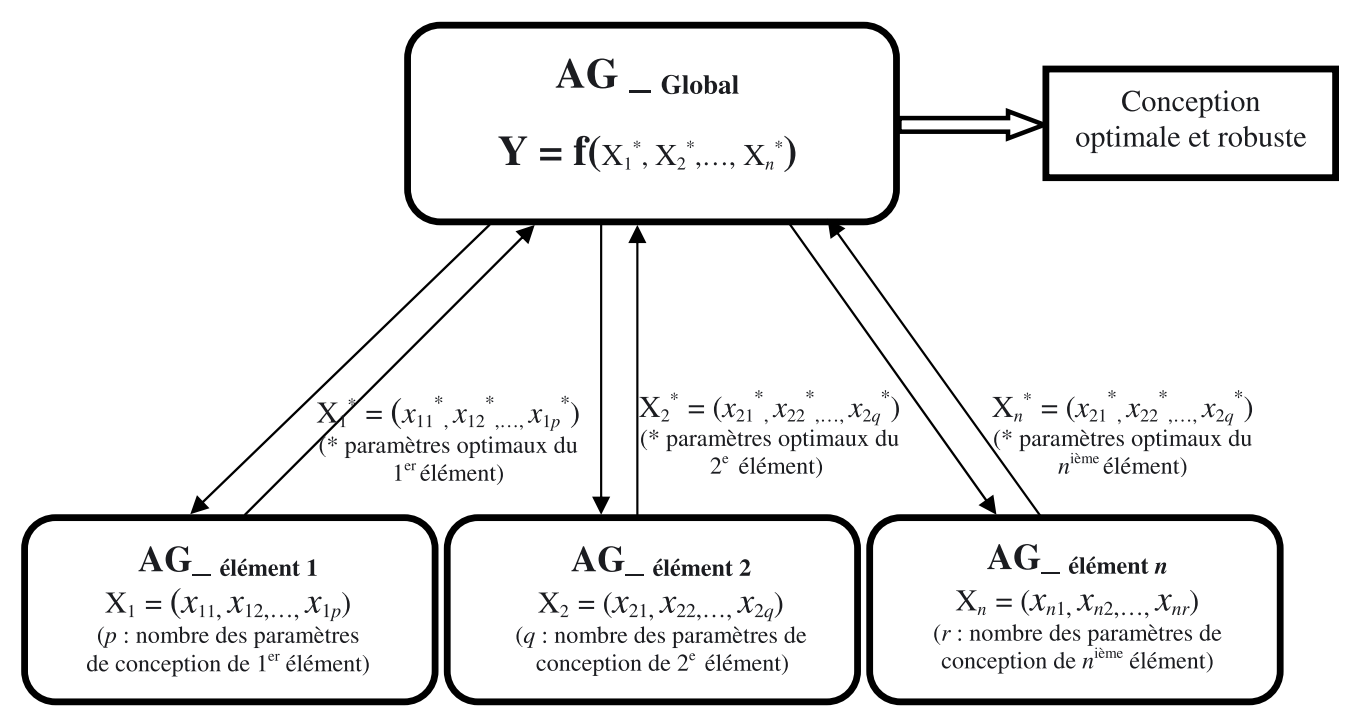

Fig. 1. Organigramme de la procédure d'optimisation proposée.

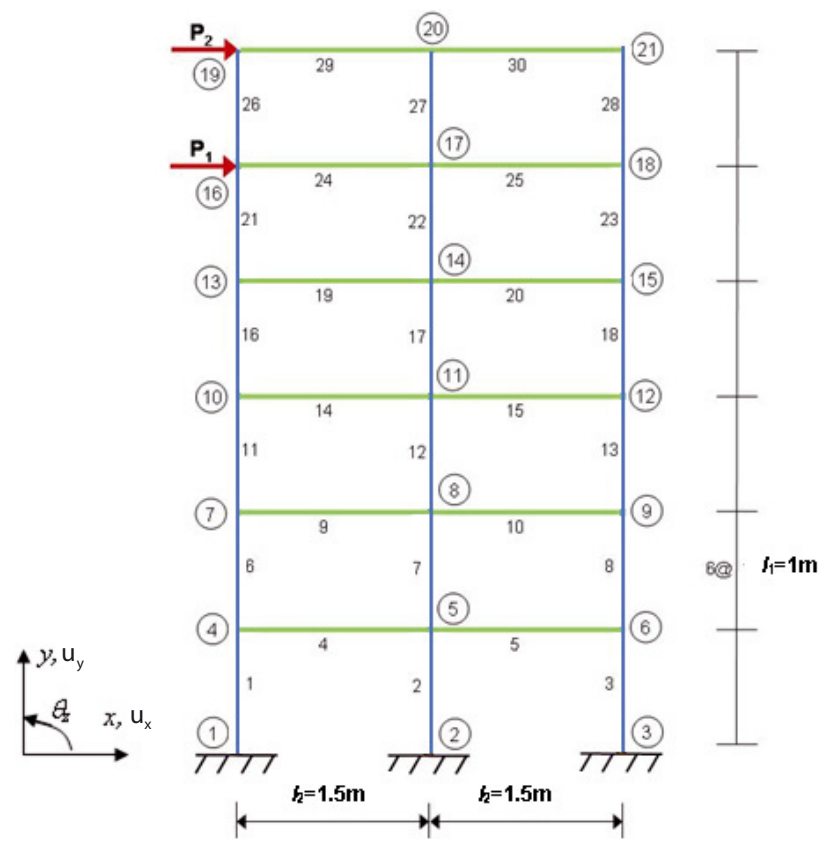

Fig. 2. Portique 2D.

\section{Application}

La structure à étudier est un portique 2D (Fig. 2). Cette structure est composée de trois poutres verticales identiques de type profilé en «I $»$ et 12 poutres horizontales identiques de type profilé en «U» (Fig. 3). Les poutres horizontales sont reliées aux poutres verticales par des liaisons soudées.

Les caractéristiques mécaniques et géométriques de la structure sont :

- la structure est homogène : le module de Young $E=2,1 \times 10^{11} \mathrm{~N} \cdot \mathrm{m}^{-2}$ et la masse volumique $\rho=7800 \mathrm{~kg} \cdot \mathrm{m}^{-3}$;
- les longueurs des poutres verticales et horizontales sont respectivement de $6 \mathrm{~m}$ et $3 \mathrm{~m}$;

- la longueur entre montants $l_{2}=1,5 \mathrm{~m}$; la hauteur d'un montant $l_{1}=1 \mathrm{~m}$;

- les montants sont des profilés en «I» (Fig. 3) de caractéristiques géométriques : $a_{1}=42 \mathrm{~mm}$; $a_{2}=70 \mathrm{~mm} ; a_{3}=6 \mathrm{~mm} ; a_{4}=4 \mathrm{~mm}$;

- les traverses (poutres horizontales) sont des profilés en « $\mathrm{U} »$ de caractéristiques géométriques : $e_{1}=$ $42 \mathrm{~mm} ; e_{2}=56 \mathrm{~mm} ; e_{3}=5 \mathrm{~mm} ; e_{4}=8 \mathrm{~mm}$.

Le MEF global de la structure comporte 54 ddl (élément fini poutre 2 nœuds et à $3 \mathrm{ddl}$ par nœud $\left.\left(u_{x}, u_{y}, \theta_{z}\right)\right)$.

- Le portique 2D est encastré aux nœuds 1, 2 et 3 (Fig. 2);

- la structure est excitée par deux forces ponctuelles synchrones et orientées horizontalement aux nœuds 16 et 19 (Fig. 2) telles que : $\boldsymbol{P}_{1}=P_{1} \cos \omega t . x$ et $\boldsymbol{P}_{2}=P_{2} \cos w t . \boldsymbol{x}$, avec $P_{1}=P_{2}=1 \mathrm{~N}$.

- on résoud dans cette étude vibratoire le problème dynamique :

$$
M \ddot{y}(t)+C \dot{y}(t)+K y(t)=P(t)
$$

où $M, C$ et $K$ sont respectivement les matrices masse, amortissement et raideur de la structure; $y$ et $P$ sont respectivement la réponse du modèle et la force harmonique appliquée.

La réponse harmonique calculée au nœud 19 provient de l'expression :

$$
y(\omega)=\left(K-\omega^{2} M+j \omega C\right)^{-1} P
$$

L'amortissement (ici de type proportionnel) peut être exprimé en fonction de la raideur par la relation $C=(\eta / \omega) K$, avec $\eta$ de l'ordre de 0,02 .

Le cahier des charges exige de minimiser simultanément la masse globale et le déplacement maximal au nœud 19 (noté $\mathrm{MaxD}_{19}$ ) de la structure globale. Afin de répondre 


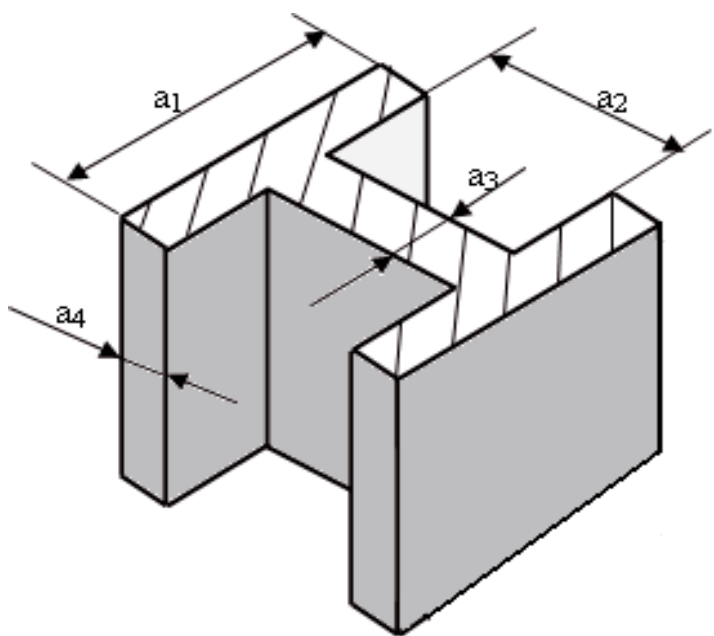

(a)

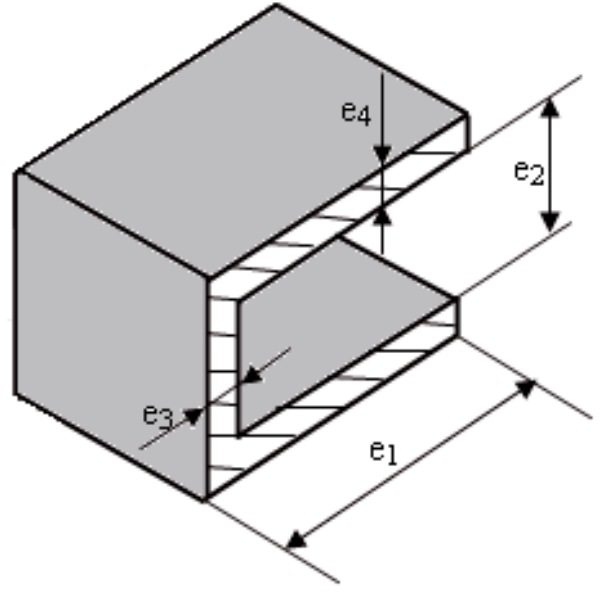

(b)

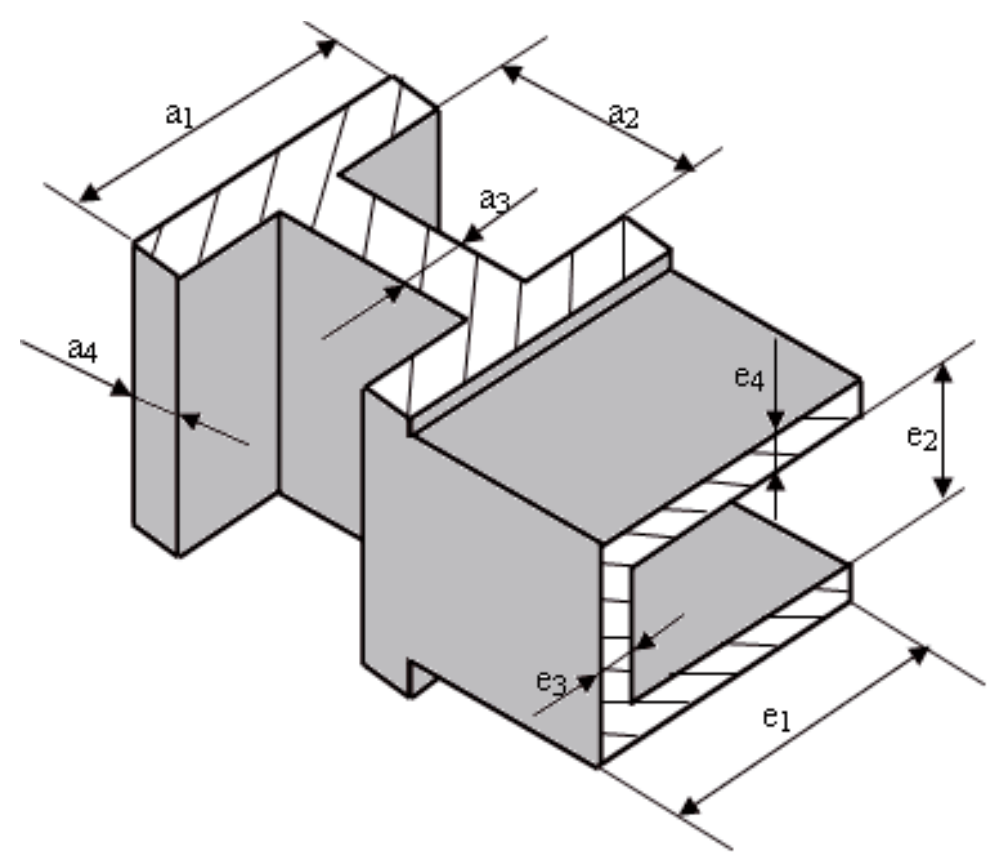

(c): $\mathrm{a}_{1}=\mathrm{e}_{1}$

Fig. 3. Section du portique : (a) profilé en $«$ I ; (b) profilé en « $\mathrm{U} » ;$ (c) connexion entre les poutres.

aux exigences de ce cahier des charges, nous avons décomposé la structure globale en deux familles de sousstructures. La première famille est composée des poutres verticales de type profilé en «I» alors que la $2^{\mathrm{e}}$ famille est formée des poutres horizontales de type profilé en $« U$. Cette décomposition est justifiée pour chaque famille d'éléments (poutres verticales ou horizontales) par la même géométrie (section, longueur...), les mêmes caractéristiques mécaniques (module d'élasticité longitudinal, masse volumique...) et les mêmes sollicitations (flexion, compression...).
La figure 4 illustre la méthodologie de décomposition en sous-structures (niveaux).

Le problème d'optimisation multi-objectifs robuste niveau élément est décomposé en deux familles d'éléments : les barres verticales et horizontales :

$1^{\text {re }}$ famille d'éléments $\quad 2^{\mathrm{e}}$ famille d'éléments

$$
\left\{\begin{array} { l } 
{ \operatorname { m i n } ( m _ { i _ { i \in 1 , 2 , 3 } } , f _ { m _ { i } } ^ { v } ) } \\
{ x \in [ a _ { 1 } , a _ { 2 } , a _ { 3 } , a _ { 4 } ] } \\
{ \text { s.c } : \sigma _ { f } \leq \sigma _ { f \operatorname { M a x } } }
\end{array} \quad \left\{\begin{array}{l}
\min \left(m_{j_{j \in 1, \ldots, 12}}, f_{m_{j}}^{v}\right) \\
x \in\left[e_{1}, e_{2}, e_{3}, e_{4}\right] \\
\text { s.c }: \sigma_{c} \leq \sigma_{c \operatorname{Max}}
\end{array}\right.\right.
$$




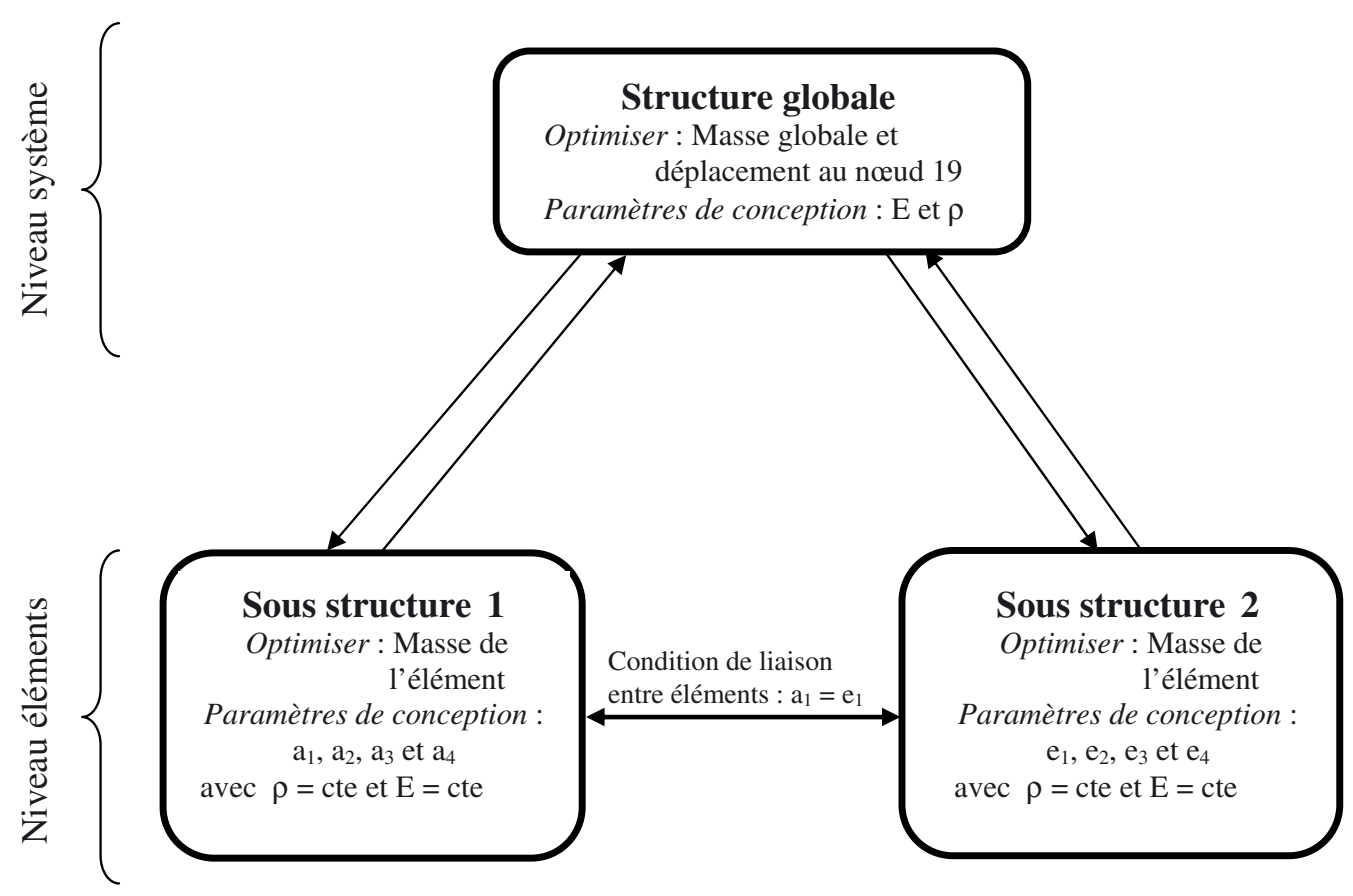

Fig. 4. Décomposition du problème multi-niveaux.

alors que le problème d'optimisation multi-objectif robuste niveau système est le suivant :

$$
\left\{\begin{array}{l}
\min \left(M_{\mathrm{g}}, f_{M_{\mathrm{g}}}^{v}, \operatorname{MaxD}_{19}, f_{\mathrm{MaxD}_{19}}^{v}\right) \\
Y \in\left[\rho, E, a_{1}^{*}, a_{2}^{*}, a_{3}^{*}, a_{4}^{*}, e_{1}^{*}, e_{2}^{*}, e_{3}^{*}, e_{4}^{*}\right] \\
\text { s.c }: a_{1}^{*}=e_{1}^{*}
\end{array}\right.
$$

où $\sigma_{\mathrm{f}}$ et $\sigma_{\mathrm{c}}$ sont respectivement des contraintes de flexion et de compression alors que $\sigma_{\mathrm{fMax}}$ et $\sigma_{\mathrm{cMax}}$ sont respectivement des contraintes maximales de flexion et de compression. $M_{\mathrm{g}}, \mathrm{MaxD}_{19}, f_{M_{\mathrm{g}}}^{v}$ et $f_{\mathrm{MaxD}_{19}}^{v}$ sont respectivement la masse globale de la structure, le déplacement maximal enregistré au nœud 19 au voisinage du mode 2 et leurs vulnérabilités. $(*)$ indique que les paramètres de conception sont optimisés au niveau élément.

On note que tous les paramètres de conception, au niveau élément et au niveau global, sont aléatoires et suivent une loi normale.

La résolution du problème d'optimisation multiobjectif robuste relatif à la $1^{\mathrm{e}}$ famille d'éléments a conduit à un nombre faible de solutions (Fig. 5). Cela est dû essentiellement à la présence de la contrainte d'une part et d'autre part à la présence de la fonction vulnérabilité. Nous avons choisi la solution qui a le meilleur compromis entre la masse et la robustesse (solution A).

Pour les mêmes raisons que précédemment, la résolution du problème d'optimisation multi-objectifs robuste relatif à la $2^{\mathrm{e}}$ famille d'éléments a aussi conduit à un nombre faible de solutions (Fig. 6). De même, le choix a été fixé sur la solution ayant le bon compromis entre la masse et la robustesse (solution B).

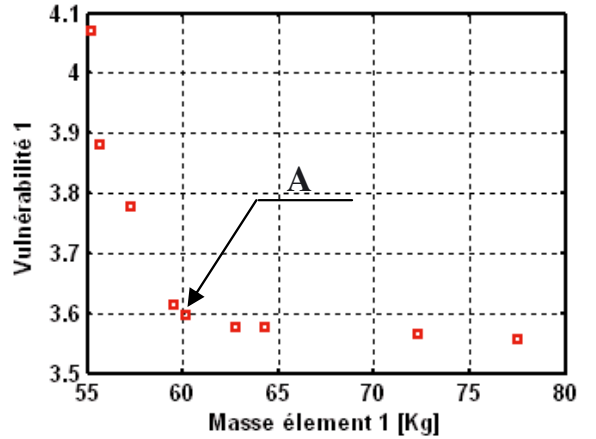

Fig. 5. Ensemble des solutions Pareto optimales robustes : $1^{\mathrm{e}}$ famille d'éléments.

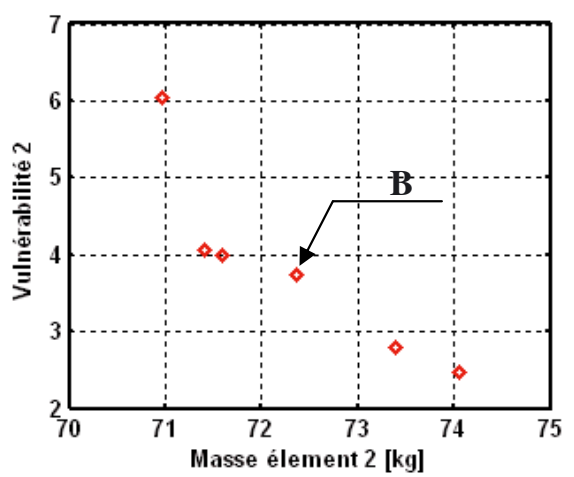

Fig. 6. Ensemble des solutions Pareto optimales robustes : $2^{\mathrm{e}}$ famille d'éléments.

En tenant compte des résultats issus de l'optimisation multi-objectifs robuste au niveau élément, le problème 

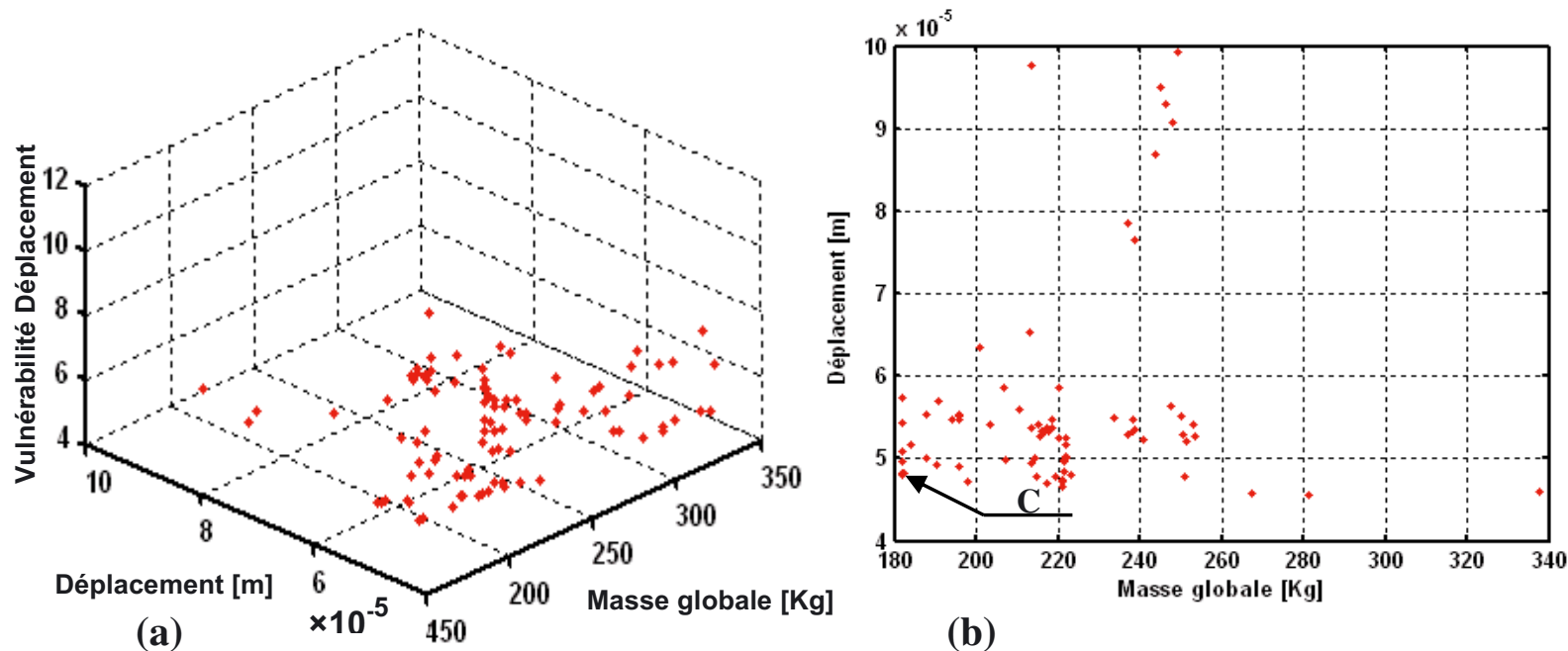

Fig. 7. (a) et (b) : ensemble des solutions Pareto optimales robustes : niveau système.

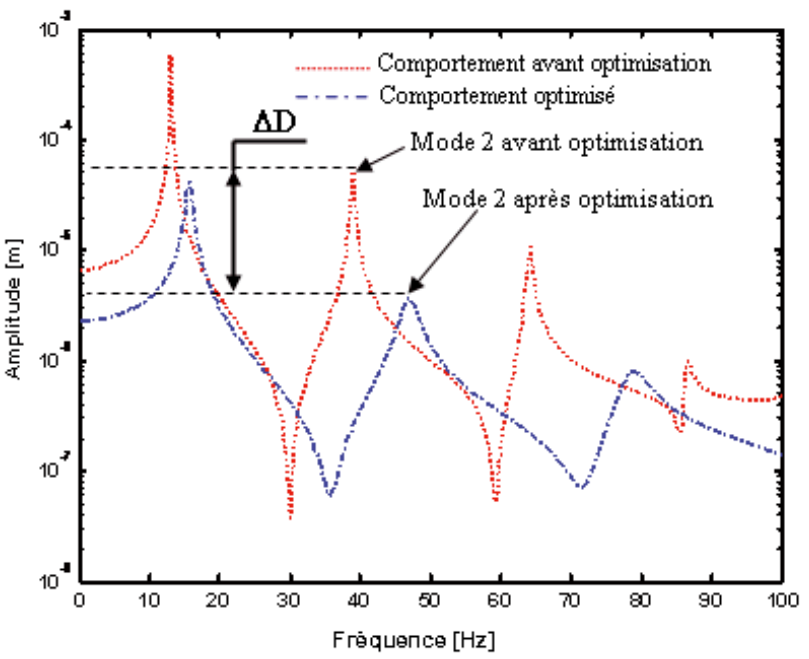

Fig. 8. Réponse forcée de la structure avant et après optimisation.

d'optimisation multi-objectifs robuste au niveau système est :

$$
\left\{\begin{array}{c}
\min \left(M_{\mathrm{g}}(Y), f_{M_{\mathrm{g}}}^{v}(Y), \operatorname{MaxD}_{19}(Y), f_{\operatorname{MaxD}_{19}}^{v}(Y)\right) \\
Y \in\left[\rho, E, a_{1}^{*}, a_{2}^{*}, a_{3}^{*}, a_{4}^{*}, e_{1}^{*}, e_{2}^{*}, e_{3}^{*}, e_{4}^{*}\right] \\
\text { avec }: a_{1}^{*}=e_{1}^{*}
\end{array}\right.
$$

Le problème à résoudre est à 4 fonctions coût, 2 paramètres de conception variables et 8 autres fixés à leurs niveaux optimaux issus du niveau élément.

La figure 7 présente l'ensemble des solutions Pareto optimales dans l'espace des fonctions coût. La figure 8 présente la réponse forcée de la structure avant et après optimisation multi-objectifs robuste multi-niveaux pour la solution C. Cette figure montre qu'avec l'application de la procédure d'optimisation proposée on a pu baisser les pics de résonance relatifs aux trois premiers modes de la bande fréquentielle $[0-100 \mathrm{~Hz}]$ et notamment celui du $2^{\mathrm{e}}$ mode (objet de notre étude) de $\Delta D \approx 90 \%$ avec un décalage fréquentiel enregistré pour tous les modes.

Afin de déterminer les performances de la méthode d'optimisation multi-niveaux (CPU + précision), une comparaison a été faite à la méthode d'optimisation mono-niveau (méthode d'optimisation multi-objectifs robuste appliquée au système global).

Le problème d'optimisation mono-niveau à résoudre est à 4 fonctions coût et 9 paramètres de conception aléatoires :

$$
\left\{\begin{array}{c}
\min \left(M_{\mathrm{g}}(Y), f_{M_{\mathrm{g}}}^{v}(Y), \operatorname{MaxD}_{19}(Y), f_{\operatorname{MaxD}_{19}}^{v}(Y)\right) \\
Y \in\left[\rho, E, a_{1}, a_{2}, a_{3}, a_{4}, e_{1}, e_{2}, e_{3}, e_{4}\right] \\
\text { avec }: a_{1}=e_{1}
\end{array}\right.
$$

La figure (9) présente l'ensemble des solutions Pareto optimales dans l'espace des fonctions coût des 2 méthodes (mono-niveau et multi-niveaux). Le tableau 1 présente les performances de la méthode proposée comparée à celle mono-niveau. On note une réduction significative en temps CPU d'environ $30 \%$ et une erreur de précision inférieur à $6 \%$. Ces performances donnent à l'ingénieur la possibilité de prendre une décision de conception rapide en phase d'avant-projet ou de projet avec une précision relativement acceptable. On note que l'erreur est déterminée par le rapport de la distance de Mahalanobis [10], calculée pour chaque nuage de solutions relatif à chaque méthode, par rapport à la référence. Cette dernière est créée par application de la méthode d'optimisation mono-niveau bruité de $1 \%$.

\section{Conclusion}

Nous avons proposé une méthode d'optimisation multi-objectifs robuste et multi-niveaux pour la conception des systèmes complexes. Cette méthode permet de 
Tableau 1. Performances de la méthode proposée.

\begin{tabular}{|c|c|c|c|}
\hline & CPU (min) & Réduction (\%) & Erreur (\%) \\
\cline { 1 - 2 } Optimisation mono-niveau & 150 & \multirow{2}{*}{30} & 6 \\
\hline Optimisation multi-niveaux & 105 & & \multirow{2}{*}{ Option } \\
\hline
\end{tabular}

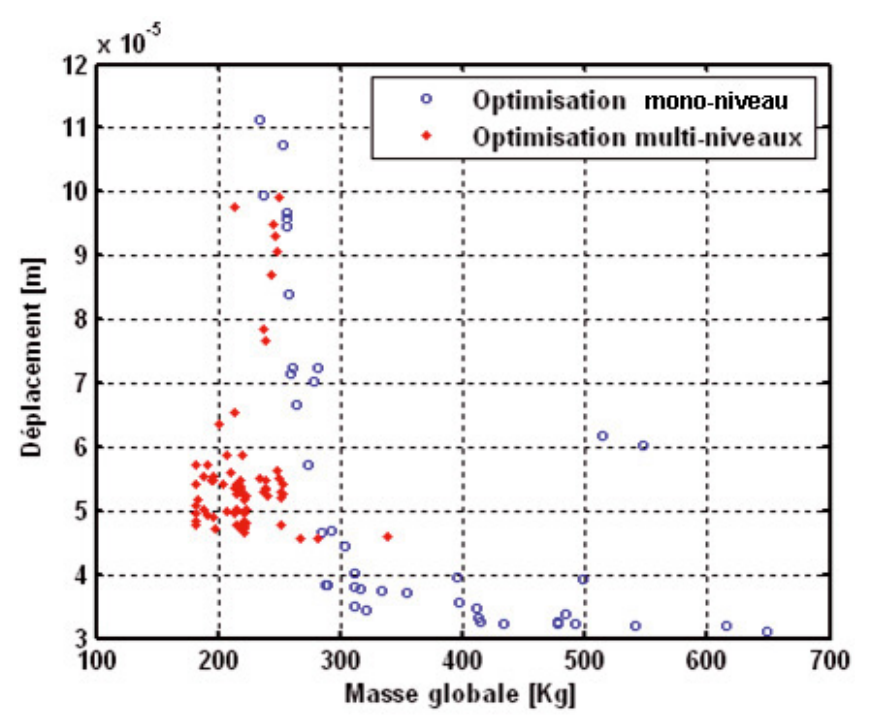

Fig. 9. Ensemble des solutions Pareto optimales robustes : optimisation mono-niveau et multi-niveaux.

gérer de manière rationnelle l'évolution des paramètres propres aux sous-systèmes et les paramètres globaux. Cette gestion peut être dictée par l'architecture du système ou par les physiques différentes dans le problème à résoudre.

Cette méthode a l'avantage de répondre au partage des tâches pratiqué dans l'industrie. De plus, elle tient compte de la présence des incertitudes sur les paramètres de conception au cours de l'optimisation, ce qui permet d'avoir, à la fin de la procédure d'optimisation, des solutions optimales et robustes vis-à-vis de ces incertitudes. L'application de cette méthode sur une structure académique a permis une réduction très significative du temps de calcul CPU par rapport à la procédure d'optimisation multi-objectifs robuste habituelle, ce qui peut en faire un outil performant d'aide à la décision en conception des structures complexes ou des systèmes multyphysiques.
Les travaux en cours concernent l'extension de l'optimisation multi-étages de structures à complexité industrielle.

\section{Références}

[1] Q.S. Li, D.K. Liu, J.Q. Fang, C.M. Tam, Multi-level optimal design of buildings with active control under winds using genetic algorithms, J. Wind Eng. Ind. Aerodyn. 86 (2000) 65-86

[2] L.U. Hansen, P. Horst, Multilevel optimization in aircraft structural design evaluation, Comput. Struct. 86 (2008) $104-118$

[3] G. Li, R.-G. Zhou, L. Duan, W.-F. Chen, Multiobjective and multilevel optimization for steel frames, Eng. Struct. 21 (1999) 519-529

[4] D. Ioannis, Z. Kang, Robust design of structures using optimization methods, Comput. Meth. Appl. Mech. Eng. 193 (2004) 2221-2237

[5] K.H. Lee, G.J. Park, Robust optimization considering tolerances of design variables, Comput. Struct. 79 (2001) $77-86$

[6] S. Ghanmi, M. Guedri, M.L. Bouazizi, N. Bouhaddi, Use of metamodels in the multi-objective optimization of mechanical structures with uncertainties, Int. J. Comput. Methods Eng. Sci. Mech. 8 (2007) 283-302

[7] N. Srinivas, K. Deb, Multi-objective optimization using nondominated sorting in genetic algorithms, Technical Report, Department of Mechanical Engineering, Institute of Technology, India, 1993

[8] B. Ait Brik, S. Ghanmi, N. Bouhaddi, S. Cogan, Robust design in structural mechanics, Int. J. Comput. Meth. Eng. Sci. Mech. 8 (2007) 33-43

[9] R.L. Iman, W.J. Conver, Small sample sensitivity analysis techniques for computer models, with an application to risk assessment, Commun. Stat. (A9) (1980) 1749-1842

[10] S. Ghanmi, M.-L. Bouazizi, N. Bouhaddi, Robustness of mechanical systems against uncertainties, Finite Elements in Analysis and Design 43 (2007) 715-731 